\title{
Pratique BIM-agile d'élicitation et de raffinement des intentions architecturales : le Micro Poker
}

\author{
Henri-Jean Gless ${ }^{1,2 *}$, Damien Hanser ${ }^{1}$, et Gilles Halin ${ }^{1,2}$ \\ ${ }^{1}$ MAP-CRAI (UMR n ${ }^{\circ}$ 3495), École Nationale Supérieure d'Architecture de Nancy, 2 rue Bastien \\ Lepage, 54000 Nancy, France \\ ${ }^{2}$ Université de Lorraine, 54000 Nancy, France
}

\begin{abstract}
Résumé. Les expérimentations présentées dans cet article viennent de l'identification puis de l'application et de la modification de pratiques agiles issues du génie logiciel au sein de la conception collaborative architecturale. En effet, la transition numérique dans le domaine de l'Architecture, Engineering and Construction (AEC) est un enjeu majeur en France et n'est souvent vue qu'à travers son aspect technologique et non humain. Notre recherche s'oriente alors vers l'apport des sciences sociales et des pratiques orientées coordination comme vecteur de changement. Les pratiques en question se présentent sous forme de jeux de cartes et permettent aux acteurs de la collaboration de mieux appréhender ce qu'est une tâche BIM et de correctement se positionner par rapport aux attentes et aux définitions des autres intervenants. Nous parlons alors de raffinement et d'élicitation des intentions architecturales.
\end{abstract}

Mots-clés. Conception architecturale, Méthodes agiles, Conception collaborative, Technologie BIM, Gestion de projet.

\begin{abstract}
The experiments presented in this article arise from the identification, the application and the modification of agile practices within collaborative architectural design. Indeed, the digital transition in the Architecture, Engineering and Construction field is a major challenge in France and is often seen only from its technological and no from its human aspect. Our research is then directed towards the contribution of social sciences and coordination-oriented practices as a vector of change. The practices are in the form of card games and enable collaboration actors to better understand what a BIM task is and to position themselves correctly in relation to the expectations and definitions of other stakeholders. We are thus talking about refinement and elicitation of architectural intentions.
\end{abstract}

Keywords. Architectural design, Agile methods, Collaborative design, BIM Technology, Project management.

${ }^{*}$ Corresponding author: gless@,crai.archi.fr 


\section{Introduction}

Notre recherche prend place dans le domaine de la conception architecturale en France, où la transition numérique est en cours, la technologie BIM n'étant toujours pas correctement implantée. De nos jours, autant dans le champ pédagogique que dans le champ professionnel, cette transition n'est souvent vue que par le prisme technologique et non pas par celui des sciences sociales.

Ainsi, nous élaborons dans le cadre de notre recherche des méthodes et des pratiques collaboratives dites agiles, terme issu du monde du génie logiciel, afin de faciliter l'adoption du BIM en conception architecturale.

Nous décrivons dans cet article des expérimentations scientifiques réalisées durant l'année 2017 dans un cadre pédagogique avec des étudiants de M2 en architecture et en Design Global. Les expérimentations consistent à introduire des pratiques agiles que nous avons adaptées pour la conception architecturale auprès de ces étudiants tout d'abord avec des exercices. Finalement, ils devront les appliquer dans un exercice concret de conception architecturale. Il s'agit pour eux de bien cerner la définition et le périmètre d'une tâche de conception BIM, afin qu'ils en aient la même compréhension. Les acteurs de la collaboration peuvent en effet avoir une approche différente, voire absente, face à la technologie BIM en raison de leurs cursus, leurs métiers ou encore leurs compétences. Nous parlons alors de raffinement des tâches de conception grâce à l'élicitation des attentes et des intentions concernant ces tâches.

Nous nous intéresserons dans la section 2 au contexte particulier de l'architecture en France. Nous verrons dans la partie 3 que notre hypothèse pour répondre aux questions qu'apporte la technologie BIM est l'adoption de pratiques agiles. Enfin, la partie 4 relatera les expérimentations réalisées en conséquence.

\section{Contexte de la recherche}

Les expérimentations présentées dans cet article se situent dans le domaine des approches collaboratives en conception architecturale et ont pour but d'améliorer le processus de conception BIM grâce à des méthodes de gestion de projet.

\subsection{Une ère de changements numériques}

Le monde de l'architecture, de l'ingénierie et de la construction (AEC) en France, plus particulièrement dans le domaine de la conception architecturale, traverse actuellement une période importante de changements; les pratiques numériques et les pratiques collaboratives sont en phase de transition et d'adaptation tandis que la technologie BIM devient à la fois une exigence réglementaire dans la construction publique et une exigence de productivité dans les projets complexes. On observe cependant une certaine inertie de la part des architectes envers le Building Information Modeling. Cette tendance peut s'expliquer à cause de la taille des agences d'architecture françaises. La plupart d'entre elles sont de petite taille ( $90 \%$ d'entre eux ont 9 employés ou moins et $75 \%$ ont 4 employés ou moins ${ }^{\dagger}$ ) et le passage au BIM représente donc un risque plus grand, mais aussi à cause du contexte socio-économique actuel, qui favorise un faible niveau d'investissement à moyen et long terme. La technologie BIM apporte également son lot de questions et de défis à relever, tant au niveau technique que de la gestion de projet.

$\dagger$ Observatoire de la profession d'architectes, Archigraphie (2016), p.48.

https://www.architectes.org/sites/default/files/atoms/files/archigraphie-coul-2016-web_0.pdf 
Ce contexte nous oriente donc vers une approche en douceur pour permettre la transition numérique, possible par la formation et les sciences sociales (Hochscheid et al., 2016).

\subsection{Des méthodes de gestion de projet émergentes}

Dans le domaine de la gestion de projet, en production ou en conception, des méthodes émergent en se réclamant de l'amélioration continue ou du Lean Production. Le Lean Construction (Dupin, 2014) qui s'applique notamment pour la gestion de chantier, conduit à une réduction des déchets et des accidents. Le Lean Management et son dérivé Last Planner permettent de responsabiliser les acteurs et de s'assurer qu'ils prennent soin les uns des autres, tout en essayant d'anticiper les besoins de chacun (Womack, 2003).

La technologie BIM augmente le volume de travail en amont (Kensek, 2014) les pratiques numériques et collaboratives et génère des malentendus, ce qui crée un climat de méfiance entre les acteurs d'un projet et rigidifie la coordination (Sakikhales, 2017).

En génie logiciel et en conception IHM, ce sont des méthodes dites agiles que l'on voit émerger depuis les années 1990 afin de répondre à ces problématiques.

La technologie BIM et ces méthodes agiles partagent des valeurs communes telles que l'application d'une meilleure communication et une réduction significative du «travail à refaire» (Beck, 2001; Kensek, 2014). Afin d'identifier les pratiques qui pourraient améliorer le processus de conception BIM, nous devons alors les expérimenter puis les adapter.

\section{Intégrer la technologie BIM grâce à l'agilité}

Les expériences que nous proposons s'inscrivent dans le cadre d'une recherche centrée sur l'identification des pratiques agiles, et leur mise en œuvre pour mesurer leurs bénéfices, d'une part dans les projets BIM des étudiants en architecture et d'autre part dans les agences d'architecture. Notre analyse pose la question de la mise en œuvre de la technologie BIM qui ne peut être résumée à de simples problèmes technologiques, il est crucial d'étudier le contexte de la mutation numérique au travers des pratiques collaboratives, de la gestion de projet et des sciences sociales.

\subsection{Le BIM complexifie les processus de conception}

Si la technologie BIM décale la quantité de travail en amont, cela est naturellement suivi par un déplacement de la communication. De plus, nouvelle technologie signifie nouveaux outils, donc nouveaux usages, et alors nouvelles pratiques (Zignale et al., 2011). Or, la technologie BIM n'est accompagnée d'aucune solution de gestion de projet.

Par ailleurs, nous avons observé divers problèmes de communication et de cohésion de groupe dans un environnement de projet BIM lors d'entretiens avec des architectes et pendant nos cours de projet : les acteurs éprouvent des difficultés à partager les résultats de leur idéation, tandis que l'utilisation de nouveaux outils BIM crée de nouvelles tâches que les acteurs ont du mal à identifier, quantifier, et à projeter sur des outils numériques (Gless et al., 2017).

Nous avons donc orienté notre travail vers des pratiques permettant aux différents acteurs d'un projet de mieux éliciter leurs intentions en identifiant et définissant

$¥$ D’après les courbes de Patrick MacLeamy. 
correctement les tâches de conception, dans le but d'améliorer la communication et la cohésion de groupe.

\subsection{Un besoin de raffinement et d'élicitation}

Les étudiants en architecture et les agences que nous rencontrons sont de plus en plus informés et sensibilisés au concept du BIM, cependant, la définition des tâches numériques reste une activité difficile à réaliser en conception; ces tâches sont mal connues, complexes, dépendent souvent d'autres tâches à effectuer par d'autres acteurs, et créent des soucis dans leur répartition (Gless et al., 2017).

Nous cherchons donc à permettre d'éliciter les intentions architecturales et à raffiner les tâches y étant associées. L'hypothèse de ce travail est que l'insertion de pratiques agiles dans les activités de conception (telle que la répartition des tâches) améliorera la communication; par l'apport d'une meilleure communication, il s'agit d'améliorer la conscience mutuelle, la prise de décision en amont, et d'aider la construction d'une confiance d'équipe : la cohésion de groupe. Une des pratiques agiles utilisées en génie logiciel pour améliorer ces aspects de la collaboration est le «Planning Poker». Son but est la construction d'un esprit d'équipe par le jeu et les discussions, en vue d'une élicitation de tous les concepteurs. Nous allons donc expérimenter cette pratique en conception architecturale.

\subsection{L'agilité comme support à la communication}

Les méthodes agiles sont des méthodes de gestion de projet qui ont émergé dans le domaine du génie logiciel et des IHM dans les années 1990. Elles impliquent davantage le client dans les chaînes de décisions et de production en réalisant de nombreuses rétrospectives et démonstrations afin d'identifier correctement ses besoins. Elles suivent également trois autres valeurs fondamentales que sont : la collaboration, l'amélioration continue et l'acceptation du changement (Beck et al., 2001). En plus de ces quatre valeurs, il existe douze principes agiles qui sont par exemple un cycle de travail régulier, encourager les conversations face à face ou faire émerger des équipes auto-organisées. Nous pouvons aussi ajouter que ces méthodes sont basées sur un cycle de production itératif, incrémental et adaptatif. Lors des cycles réguliers, un modèle évolue par incréments, et ce en fonction des retours du client.

Comme la technologie BIM, l'agilité mise sur une meilleure communication et une meilleure coordination, et permet aux différents acteurs de correctement se positionner par rapport aux idées et aux intentions individuelles de l'équipe (l'élicitation) et de mieux comprendre ce qu'ils doivent faire (le raffinement).

\subsection{Le Planning Poker comme pratique élicitative}

Le Planning Poker est une pratique agile popularisée par Mike Cohn (Cohn, 2005). Il s'agit d'un serious game permettant de comparer et d'évaluer des scénarios de fonctionnalités à développer en informatique. Les différents acteurs d'un projet (ici les concepteurs logiciel) disposent chacun d'un jeu d'une dizaine de cartes numérotées de 1 à 100 en suivant la croissance d'une courbe de Fibonacci arrondie (1, 2, 3, 5, 8, 13, 20, 40 et 100). Ils s'en servent alors pour estimer la complexité ou la durée d'une fonctionnalité. Pour ce faire, on énonce une fonctionnalité (une liste peut être préparée par un meneur de jeu par exemple), puis on se demande par exemple en combien d'heures cette dernière peut être réalisée. Chaque joueur choisit une carte dans sa main (la carte 1 représentant alors une heure). Les joueurs déposent simultanément devant eux la carte choisie. S'en suit un débat 
entre le joueur avec la plus grande estimation et celui ayant la plus petite. Au fur et à mesure, les autres joueurs peuvent donner leur avis jusqu'à ce qu'on arrive à un compromis donnant lieu à une évaluation commune et validée par tous. Ce protocole de prise de parole permet d'éviter le phénomène du «premier parleur», qui fait que les autres joueurs baseraient alors leurs estimations d'après celles de la première personne à s'exprimer.

\section{Expérimentation de pratiques agiles en conception architecturale}

Le Planning Poker a été testé lors d'un exercice sur un court projet architectural auprès d'étudiants de M2 en architecture et en Design Global. Après avoir expérimenté la version classique du jeu, ils ont joué à des versions adaptées pour l'architecture lors d'exercices sur une journée puis dans un projet long à l'échelle d'un semestre.

\subsection{Protocole expérimental}

Dès le lancement de l'année universitaire, nous avons suivi ce protocole, constitué de plusieurs phases :

- Phase théorique : cours d'introduction sur l'agilité et la gestion de projet suivi d'une explication du Planning Poker;

- Phase pratique : exercice court de Planning Poker;

- Récolte de données : les étudiants notent et nous rendent leurs estimations et les temps réels mesurés ;

- Analyse des données : amélioration de la pratique (ici en Micro Poker);

- Phase pratique : exercice court de Micro Poker;

- Phase pratique : projet architectural BIM sur un semestre ;

- Récolte de données : soutenance orale à propos de la collaboration dans le précédent projet, puis diffusion d'un questionnaire à propos de la pratique et des méthodes agiles en général ;

- Analyse des données : tris des biais et amélioration de la pratique.

Les phases théoriques permettent aux étudiants de comprendre les enjeux de la gestion de projet en architecture tout en faisant une introduction aux méthodes et pratiques agiles.

La première phase pratique consiste en un exercice de la pratique traditionnelle du Planning Poker. La seconde concerne quant à elle notre version modifiée du Micro Poker. Enfin, la dernière permet d'appliquer cette pratique à un projet traditionnel de conception architecturale dans un environnement BIM.

Les phases de récolte de données nous permettent de recevoir en complément de nos observations lors des phases pratiques, des retours soit purement quantitatifs grâce aux retours des estimations des étudiants, soit qualitatifs avec l'organisation d'une soutenance suite au projet ainsi que la distribution d'un questionnaire anonyme.

Enfin, les phases d'analyse de données nous permettent de vérifier nos hypothèses quant à l'amélioration de la communication et de la cohésion après le tri de biais (réponses non pertinentes dans le questionnaire, etc.). La première s'est logiquement suivie de l'adaptation du Planning Poker en Micro Poker (voir 4.3 Adaptation vers le Micro Poker). 


\subsection{Expérimentation du Planning Poker}

Durant un exercice court de projet de conception architecturale, des étudiants de M2 ont expérimenté le Planning Poker. Les étudiants venaient de cursus différents : architecture, ingénierie et design. L'exercice consistait en la conception d'un abri. En une journée, ils devaient par groupe de quatre réaliser cinq tâches : 1) importer un nuage de points dans un logiciel BIM, 2) le placer, 3) modéliser l'existant de leur terrain, 4) modéliser l'abri, puis 5) exporter le tout pour un autre cours.

Afin d'estimer et de bien identifier ces tâches, chaque groupe d'étudiants devait utiliser le Planning Poker. Les résultats du groupe 1 sont présentés ci-après (cf. figure 1).

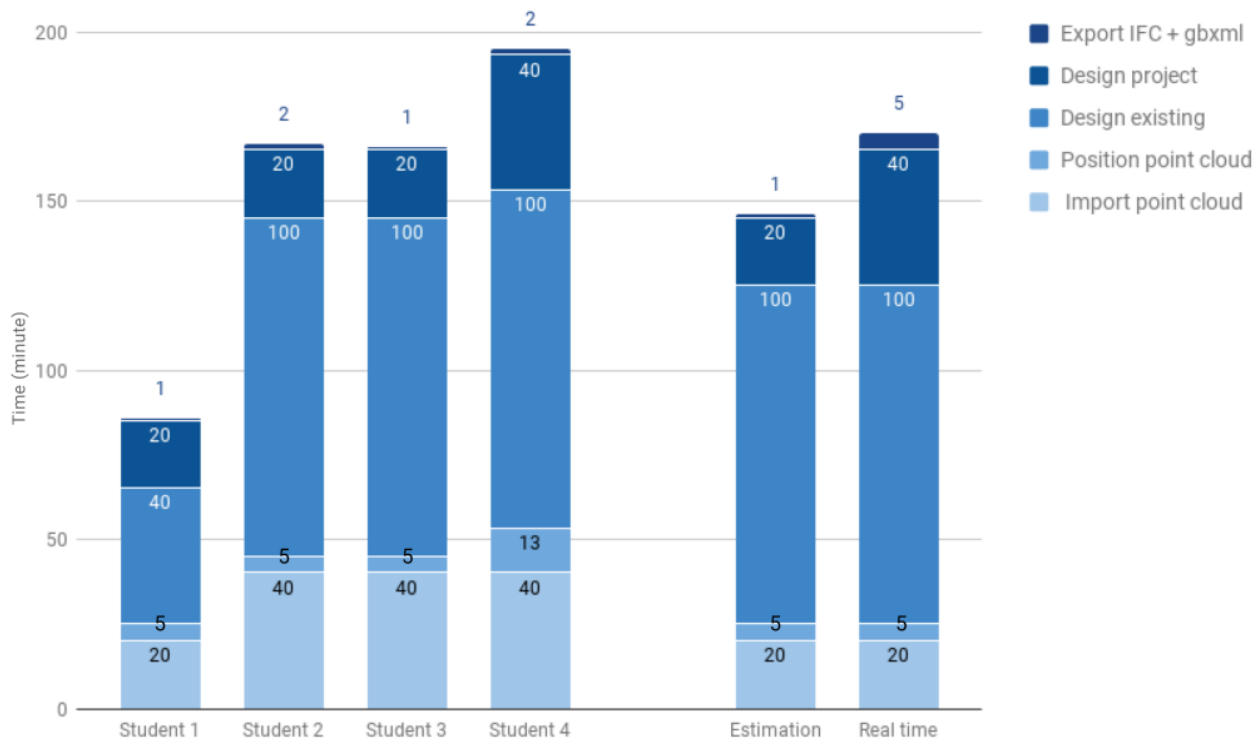

Figure 1. Résultats d'une séance de Planning Poker pour un groupe : estimations personnelles, estimation négociée et temps réel.

On observe ainsi que l'étudiant 1 avait des estimations de durées inférieures au temps réel pour la modélisation de l'existant. Ces résultats s'expliquent parce que les trois autres étudiants avaient des connaissances plus poussées dans le domaine de la modélisation numérique et savaient mieux estimer les durées associées.

Ainsi, il faut garder en tête que ce qui nous intéresse dans le Planning Poker est son aspect bénéfique sur le plan de la communication et de la cohésion du groupe : tous les joueurs s'expriment et font face à la perception que les autres ont des tâches à accomplir. Même si les estimations sont fausses, les étudiants ont eu une meilleure vue d'ensemble des choses à faire et des intentions de chacun.

Cette expérimentation a montré que les estimations de temps données par les groupes étaient cohérentes avec le temps réel pour accomplir l'exercice. Néanmoins, les étudiants avaient du mal à s'approprier le jeu du fait de son grand nombre de cartes (Gless et al., 2017). Par ailleurs, les étudiants se posaient également des questions sur la priorité des tâches, ou avaient du mal à estimer une complexité avec des nombres. Ils pouvaient par ailleurs ne pas tomber d'accord suite à un débat. 


\subsection{Adaptation vers le Micro Poker}

En réponse à ces remarques, nous avons mis au point une variante du Planning Poker, intitulée le Micro Poker. Au départ, un jeu de seulement trois cartes, il s'agit désormais d'un jeu de quatre cartes (la carte du milieu posait des problèmes de «non-choix » de la part des joueurs, en se positionnant au centre). Nous avons alors non seulement des estimations sur une échelle numérique, mais également sur trois autres : une échelle de grandeur, de couleur, et de hasard. Les règles restent les mêmes. Les cartes portent les valeurs suivantes (cf. figure 2) :

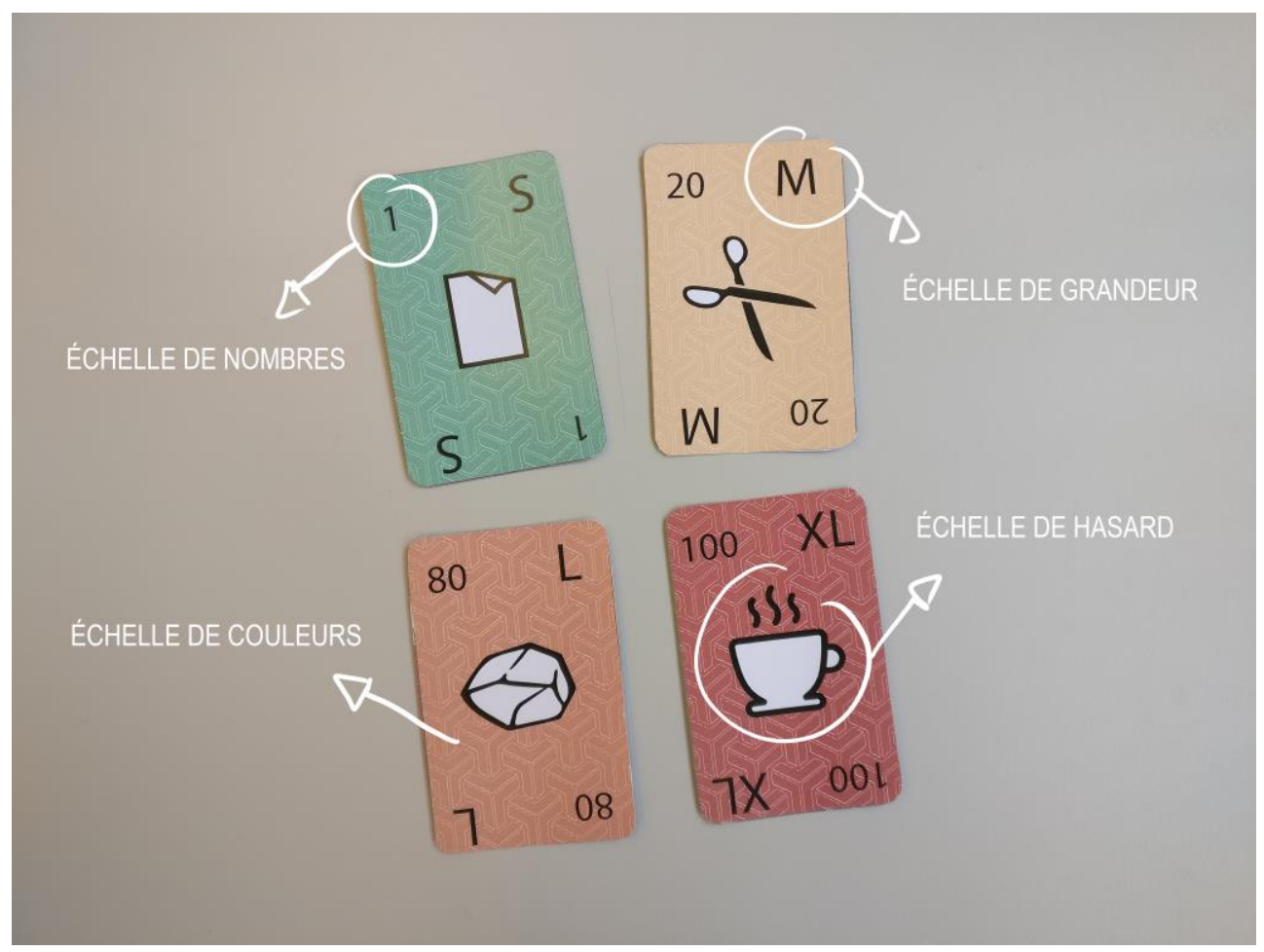

Figure 2. Les cartes de la version 2 du Micro Poker.

- Numérique : 1, 20, 80 et 100 pouvant estimer un temps en valeur relative $(10 \%$ ou $80 \%$ de la journée); il s'agit ici de simplifier le choix de la carte en le limitant aux extrêmes et à deux valeurs intermédiaires ;

- Grandeur : S, M, L et XL pouvant représenter la quantité de travail à faire ; parfois, les nombres ne peuvent permettre de définir une valeur, d'où des « tailles »;

- Couleur : vert, jaune, orange et rouge, pouvant représenter l'urgence d'une activité ou l'approbation d'une décision (le rouge étant alors très urgent ou négatif, contrairement au vert) ;

- Hasard : si un débat ne peut être clos, les cartes pierre, feuille et ciseau permettent d'invoquer le hasard, tandis que la carte café permet de demander une pause.

Afin de choisir quelle échelle d'évaluation choisir, le meneur de jeu se pose simplement la question suivante: «ce que nous allons évaluer s'inscrit-il dans une échelle de temps 
(nombres), dans une échelle de grandeur (tailles) ou dans une échelle d'urgence (couleurs) ?».

Tout comme le Planning Poker classique, il s'agit ici d'un support à la communication qui permet l'élicitation et le raffinement des tâches de conception. Même si l'estimation est fausse, le but est de parler. Les cartes plus colorées et avec des pictogrammes renforcent également le côté ludique de la pratique, pour que les acteurs se prennent plus facilement au jeu.

\subsection{Expérimentation du Micro Poker}

Lors d'un atelier de projet se déroulant sur huit semaines, les étudiants du Master 2 de la promotion suivante ont expérimenté le Micro Poker. Ils devaient concevoir une caserne de pompiers dans un contexte de collaboration BIM. Les étudiants venaient toujours de cursus différents, et le cours se déroulant en début de semestre, les groupes ont été constitués pour que les étudiants ne se connaissent pas. De plus, les tâches à effectuer ici n'étaient pas données, les étudiants étant libres de travailler comme ils le voulaient. Voici l'exemple d'un échange entre étudiants (cf. figure 3 ) :

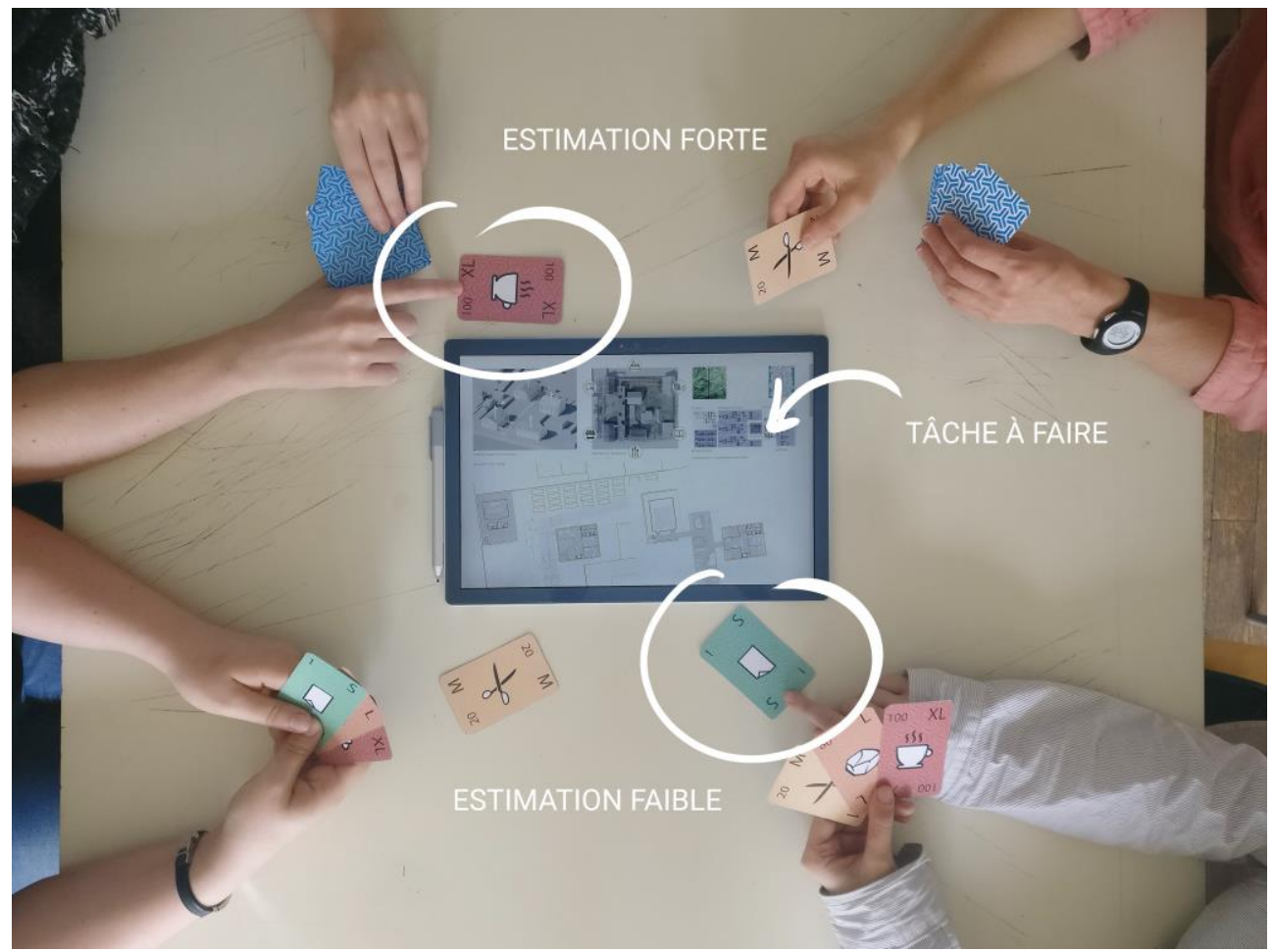

Figure 3. Illustration d'un échange de Micro Poker

- Étudiant 1 : y a-t-il beaucoup de travail pour modéliser le terrain ? On utilise les cartes $\mathrm{S}, \mathrm{M}, \mathrm{L}$ et XL (tout le monde choisit une carte, et la révèle en même temps ; étudiants 1 et 2 ont choisi respectivement $\mathrm{S}$ et XL et commencent donc à parler)

- Étudiant 1 : j'ai choisi S car ce n'est pas difficile de modéliser un terrain. 
- Étudiant 2 : moi j'ai pris XL car on n'a pas le cadastre complet de la parcelle. On doit chercher les courbes de niveau aussi.

- Étudiant $1:$ ah ! Je n'avais pas pensé à ça.

- Étudiant 3 : et on peut en profiter pour aller faire des relevés. Je crois que le bâtiment sur la vue aérienne n'existe plus.

- Étudiant 1 : ah oui. Eh bien, je m'occupe de trouver tout ce qu'il faut pour modéliser et je commence ; je maîtrise bien ArchiCad.

- Étudiant 2 : ok, avec Étudiant 3 on va aller sur le terrain alors.

- Étudiant 1 : bien, du coup question suivante. Y a-t-il beaucoup de travail pour dessiner le parking demandé ? (Ici commence un nouveau tour.)

On observe ici que le Micro Poker a permis aux étudiants de confronter les idées qu'ils avaient de la tâche de modélisation quant à sa définition. Cette dernière comportait plus ou moins de sous-tâches selon les acteurs. Cela leur a donc permis non pas forcément d'évaluer avec justesse le travail à faire, mais tout du moins à mieux en dessiner les contours.

\subsection{Résultats}

La pratique du Micro Poker a été perçue de manière plutôt positive par les étudiants (voir figure. 4). Il permet d'augmenter le nombre d'échanges dans un groupe de travail, et de discuter de la quantité de travail qu'ils ont à produire, de la manière dont ils vont collaborer, de qui réalise quoi, etc. Le questionnaire proposé à la fin du semestre à propos des pratiques expérimentées donne les réponses suivantes :

Pensez-vous que le Micro Poker vous aura permis de mieux communiquer?

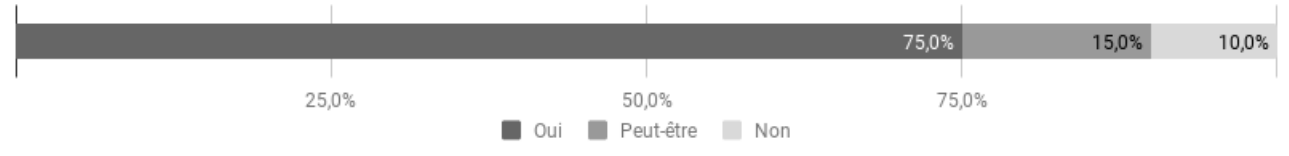

Pensez-vous réutiliser des pratiques agiles dans vos projets futurs?
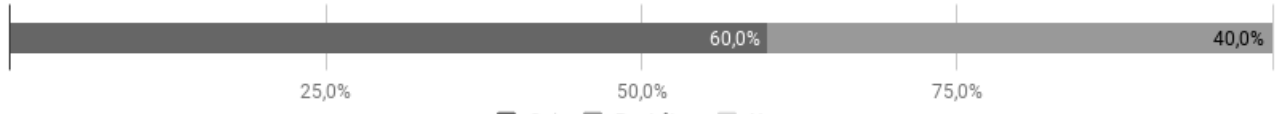

Oui $\square$ Peut-être Non

Figure 4. Résultats du questionnaire sur le Micro Poker et les pratiques agiles en général.

Nous pouvons cependant émettre une réserve. Les étudiants avaient tendance à utiliser les cartes comme un système de vote grâce aux couleurs verte et rouge, ou bien à proposer quatre solutions et les attribuer à chacune des cartes avant de voter, ce qui constitue en soi une pratique différente.

\section{Conclusion et perspectives}

Ces premières expériences montrent que le Micro Poker permet de mieux dessiner les contours d'une tâche de conception architecturale et d'en valider la définition, ainsi que permettre une meilleure communication et cohésion de groupe. Les tenants et les aboutissants définissant une tâche ne dépendant alors plus de l'idée que chacun se fait de la tâche et de l'action même de collaborer, mais d'une discussion argumentée et collégiale de 
la part de tous les acteurs de la conception. Le Micro Poker sensibilise également à l'agilité par son côté ludique.

Par ailleurs, nous pouvons noter que les étudiants se sont pour la plupart approprié le Micro Poker grâce à son aspect ludique. En effet, certains groupes s'en servaient afin de choisir des activités en dehors des expérimentations, tel que leur lieu de repas. De la même manière, les enseignants du semestre s'en sont également servi afin d'évaluer les résultats des étudiants de manière non biaisée, en les classant avec les cartes plutôt qu'en attribuant des notes.

Afin de valider définitivement les apports de cette pratique agile, nous réaliserons avec les étudiants une dernière expérimentation, conscrite cette fois sur une durée d'une semaine et dans laquelle nous introduirons d'autres pratiques agiles.

Enfin, nous allons tester dans les semaines à venir le Micro Poker dans des agences d'architecture. C'est une première étape dans l'intégration du BIM car cette pratique nous permet de définir une tâche mal connue : une tâche BIM.

\section{Bibliographie}

Beck, K. et al., (2001). Manifesto for agile software development. http://agilemanifesto.org Cohn, M. (2005). Agile Estimating and Planning. Prentice Hall ; 1ère édition. ISBN-13 : 978-0131479418.

Dupin, P., (2014). Le LEAN appliqué à la construction : Comment optimiser la gestion de projet et réduire coûts et délais dans le bâtiment? Éditeur : Eyrolles.

Gless, H.-J. and al., (2017). BIM-agile practices experiments in architectural design. In : Luo Y. (eds) Cooperative Design, Visualization, and Engineering. CDVE 2017. Lecture Notes in Computer Science, vol 10451, pp. 135-142. Springer, Cham.

Hochscheid, E. et al., (2016). Intégration de pratiques BIM en agences. SCAN'16 Toulouse. Presses Universitaires Nancy.

Kensek, K. et al., (2015). Manuel BIM : Théories et Applications. Eyrolles.

Sakikhales, M., (2017). Using Agile Project Management and BIM for Improved Building Performance. BIM, Building Performance, Design and Smart Construction Projects.

doi : 10.1007/978-3-319-50346-2_5.

Womack, J., Jones, D., (2003). Lean Thinking: Banish Waste and Create Wealth in Your Corporation, Revised and Updated. Éditeur: Free Press ; Édition : 2 (10 juin 2003) ISBN 978-0743249270.

Zignale, D. and al., (2011). Modelling practices and usages to improve adaptation of group-ware-tool services : Application in the AEC sector. EuropIA.13 : 13th International Conference on Advances in Design Sciences and Technology. Roma, Italy. 